\title{
NO EXPANDING FIREBALL: RESOLVING THE RECURRENT NOVA RS OPHIUCHI WITH INFRARED INTERFEROMETRY
}

\author{
J. D. Monnier, ${ }^{1}$ R. K. Barry, ${ }^{2,3}$ W. A. Traub,${ }^{4,5}$ B. F. Lane, ${ }^{6}$ R. L. Akeson, ${ }^{7}$ S. Ragland,${ }^{8}$ P. A. Schuller,${ }^{4}$ \\ H. Le Coroller, ${ }^{9}$ J.-P. Berger, ${ }^{10}$ R. Millan-Gabet, ${ }^{7}$ E. Pedretti, ${ }^{1}$ F. P. Schloerb,${ }^{11}$ C. Koresko, ${ }^{7}$ N. P. Carleton, ${ }^{4}$ \\ M. G. Lacasse, ${ }^{4}$ P. Kern,${ }^{10}$ F. Malbet,${ }^{10}$ K. Perraut, ${ }^{10}$ M. J. Kuchner, ${ }^{3}$ and M. W. Muterspaugh ${ }^{12}$ \\ Received 2006 May 22; accepted 2006 July 11; published 2006 August 8
}

\begin{abstract}
Following the recent outburst of the recurrent nova RS Oph on 2006 February 12, we measured its near-infrared size using the IOTA, Keck, and PTI Interferometers at multiple epochs. The characteristic size of $\sim 3$ mas hardly changed over the first 60 days of the outburst, ruling out currently popular models whereby the near-infrared emission arises from hot gas in the expanding shock. The emission was also found to be significantly asymmetric, evidenced by nonzero closure phases detected by IOTA. The physical interpretation of these data depends strongly on the adopted distance to RS Oph. Our data can be interpreted as the first direct detection of the underlying RS Oph binary, lending support to the recent "reborn red giant" models of Hachisu \& Kato. However, this result hinges on an RS Oph distance of $\lesssim 540 \mathrm{pc}$, in strong disagreement with the widely adopted distance of $\sim 1.6 \mathrm{kpc}$. At the farther distance, our observations imply instead the existence of a nonexpanding, dense, and ionized circumbinary gaseous disk or reservoir responsible for the bulk of the near-infrared emission. Longer baseline infrared interferometry is uniquely suited to distinguish between these models and to ultimately determine the distance, binary orbit, and component masses for RS Oph, one of the closest known (candidate) Type 1a supernova progenitor systems.
\end{abstract}

Subject headings: infrared: stars — novae, cataclysmic variables — stars: individual (RS Ophiuchi) — techniques: interferometric

\section{INTRODUCTION}

Most astronomers are familiar with classical novae, exploding stars in which an accreting white dwarf (WD) in an interacting binary system accumulates enough material for it to become unstable to hydrogen burning. The expanding blast wave from one such event Nova Aql 2005 was recently observed by infrared (IR) interferometry (Lane et al. 2005), and the geometric distance was estimated based on velocities from spectral line observations. This result is consistent with the "optically thick fireball" model, which has been successfully used for 20 years to explain the time evolution of the spectral energy distribution (SED) of classical novae (Gehrz 1988).

While classical novae are expected to recur, very few actually have in recorded history. RS Oph is one of the handful of socalled recurrent novae with (now) six outbursts since 1898 (Warner 1976). The most recent outburst occurred on 2006 February 12 (Narumi et al. 2006), and this unusual event motivated intense monitoring by the IR interferometry community.

\footnotetext{
${ }^{1}$ University of Michigan Astronomy Department, 941 Dennison Building, Ann Arbor, MI 48109-1090; monnier@umich.edu.

${ }^{2}$ Johns Hopkins University, 3400 North Charles Street, Baltimore, MD 21218.

${ }^{3}$ NASA Goddard Space Flight Center, Greenbelt, MD 20771.

${ }^{4}$ Harvard-Smithsonian Center for Astrophysics, MS 20, 60 Garden Street, Cambridge, MA 02138.

${ }^{5}$ Jet Propulsion Laboratory, California Institute of Technology, 4800 Oak Grove Drive, Pasadena, CA 91109.

${ }^{6}$ Massachusetts Institute of Technology, 77 Massachusetts Avenue, Cambridge, MA 02139.

${ }^{7}$ Michelson Science Center, California Institute of Technology, Pasadena, CA 91125.

${ }^{8}$ W. M. Keck Observatory, California Association for Research in Astronomy, Kamuela, HI 96743.

${ }^{9}$ Observatoire de Haute-Provence (CNRS/OAMP), F-04870 Saint Michel l'Observatoire, France.

${ }^{10}$ Laboratoire d'Astrophysique de Grenoble, CNRS, Université Joseph Fourier, B.P. 53, F-38041 Grenoble, France.

${ }^{11}$ University of Massachusetts, Amherst, MA 01003.

${ }^{12}$ California Institute of Technology, Pasadena, CA 91125.
}

The special nature of RS Oph is thought to stem from two causes. First, the WD is likely extremely close to the Chandrasekhar limit, since the amount of hydrogen needed to trigger an outburst decreases dramatically as the WD mass increases. Indeed, detailed models indicate the WD mass is within $1 \%$ of exploding as a Type Ia supernova (SN Ia; e.g., Hachisu \& Kato 2001). Second, the mass-losing companion for RS Oph is a red giant $(\mathrm{RG})$ with a wind, providing a high-density medium for accretion onto the WD as well as for the exploding blast wave to interact with. Bode \& Kahn (1985) have produced the most successful model for recurrent novae, drawing a clear analogy to extragalactic supernovae and explaining the radio and X-ray light curves in this context.

Evans et al. (1988) were the first to study in detail the IR time evolution of a recurrent nova. They monitored closely the 1-3.5 $\mu \mathrm{m}$ flux of RS Oph for about 3 years after the 1985 eruption. They found that the light curve had a characteristic (2 mag) decay timescale of about 30 days and compared their observations to the generic predictions of the Bode \& Kahn (1985) model. They concluded that their observations could come from the hot, postshock gas; this model would predict that the IR source should be seen linearly expanding at a rate of about $\sim 1$ mas day $^{-1}$ at a distance of $1.6 \mathrm{kpc}$. This distance estimate is based (most securely) on the expanding size of the radio emission observed in 1985 by Hjellming et al. (1986) and Taylor et al. (1989), assuming association with the forward shock (new radio data reconfirm the 1985 observations; M. Rupen, A. J. Mioduszewski, \& M. Sokoloski 2006, in preparation; O'Brien et al. 2006). As will become clear, the distance to RS Oph is key to the interpretation of the IR interferometry data presented here.

Challenging this interpretation, Hachisu \& Kato (2001; see also Kato 1991) have recently produced a comprehensive model for recurrent novae meant to explain a wide range of the known nova properties, and it makes a specific prediction for the origin of the near-infrared (NIR) continuum that is very 
TABLE 1

OBSERVING LOG FOR RS OPH

\begin{tabular}{|c|c|c|c|}
\hline $\begin{array}{c}\text { Days Since } \\
2006 \text { February } 12\end{array}$ & $\begin{array}{l}\text { Date } \\
\text { (UT) }\end{array}$ & $\begin{array}{l}\text { Interferometer } \\
\text { (Configuration) }\end{array}$ & $\begin{array}{l}\text { Wavelength } \\
(\mu \mathrm{m})\end{array}$ \\
\hline \multirow[t]{2}{*}{4 .............. } & 2006 Feb 16 & IOTA $(\mathrm{A} 20 \mathrm{~B} 15 \mathrm{C} 00)^{\mathrm{a}}$ & 1.65 \\
\hline & 2006 Feb 16 & $\mathrm{Keck}^{\mathrm{b}}$ & 2.18 \\
\hline 11 & 2006 Feb 23 & IOTA (A20B15C00) & 1.65 \\
\hline 14 & 2006 Feb 26 & IOTA (A35B15C10) & 1.65 \\
\hline 19 & 2006 Mar 03 & IOTA (A35B15C10) & 1.65 \\
\hline 20 & 2006 Mar 04 & IOTA (A35B15C10) & 1.65 \\
\hline 22 & 2006 Mar 06 & IOTA (A35B15C10) & 1.65 \\
\hline 25 & 2006 Mar 09 & IOTA (A35B15C10) & 1.65 \\
\hline 29 & 2006 Mar 13 & IOTA (A35B15C10) & 1.65 \\
\hline 49 & 2006 Apr 02 & Palomar Testbed $(\mathrm{NW})^{\mathrm{c}}$ & 2.20 \\
\hline 59 & 2006 Apr 12 & IOTA (A35B15C10) & 1.65 \\
\hline 60 & 2006 Apr 13 & IOTA (A35B15C10) & 1.65 \\
\hline 63 & 2006 Apr 16 & Palomar Testbed (NW) & 2.20 \\
\hline 65 & 2006 Apr 18 & Palomar Testbed (NW) & 2.20 \\
\hline
\end{tabular}

Note.-Uniform disk (UD) diameters of calibrators were generally estimated using getCal, an SED-fitting routine maintained and distributed by the Michelson Science Center (http://msc.caltech.edu).

${ }^{a}$ IOTA used the following calibrators: HD 152601 (1.6 \pm 0.4 mas), HD 164064 (1.6 \pm 0.5 mas), $\chi$ UMa (3.24 \pm 0.04 mas; Bordé et al. 2002), $\rho$ Boo (3.72 \pm 0.12 mas; van Belle et al. 1999), HD 143033 (1.9 \pm 1.5 mas), HD 156826 (0.6 \pm 0.2 mas), HD 157262 ( $1.5 \pm 0.5$ mas).

${ }^{\mathrm{b}} \mathrm{KI}$ used the following calibrators: $\chi \mathrm{UMa}(3.35 \pm 0.17$ mas; Cohen et al. 1999), $\rho$ Boo (3.92 \pm 0.19 mas; Cohen et al. 1999).

${ }^{c}$ PTI used the following calibrators: HD 164064 (1.6 \pm 0.5 mas), HD 161868 ( $0.7 \pm 0.1$ mas $)$.

different from Evans et al. (1988). Following onset of the thermonuclear runaway of the hydrogen shell around the WD, the shell expands to AU size, in effect turning the WD back into a red giant. The shell stably burns hydrogen for a few weeks, shrinking back to the size of white dwarf. According to this model, hot postshock gas plays no role in forming the IR continuum. Furthermore, Hachisu \& Kato (2001) prefer a much closer distance of $600 \mathrm{pc}$, implying a binary separation of 2.9 mas, easily detectable with current interferometers.

In this Letter, we report first-ever size measurements for RS Oph in the NIR using long-baseline interferometry. Our results are surprising, ruling out the favored expanding fireball model, raising doubts about the established distance to RS Oph, and motivating a new model for the NIR emission.

\section{OBSERVATIONS}

In this Letter we report on data from three different interferometers; a summary of observations can be found in Table 1. Here we briefly introduce each data set.

Most of our data were obtained at the Infrared-Optical Telescope Array (IOTA; Traub et al. 2003), which has baselines between 5 and $38 \mathrm{~m}$. The IONIC3 combiner (Berger et al. 2003) was used to measure three visibilities $\left(V^{2}\right)$ and one closure phase (CP) simultaneously in the broadband $H$-band filter $\left(\lambda_{0}=1.65 \mu \mathrm{m}\right.$, $\Delta \lambda=0.3 \mu \mathrm{m})$. Data analysis procedures have been documented in recent papers (Monnier et al. 2004, 2006). For the data here, we have adopted a calibration error $\Delta V^{2}=0.05$ (relative error) on baselines $\mathrm{AB}$ and $\mathrm{AC}$ and $\Delta V^{2}=0.10$ for baseline $\mathrm{BC}$.

The Keck Interferometer (KI) was used to observe RS Oph on a single night 4 days after the burst (UT 2006 February 16) with a baseline of $\sim 85 \mathrm{~m}$. Facility and instrument descriptions can be found in recent KI publications (Colavita et al. 2003; Colavita \& Wizinowich 2000, 2003). The $K$-band $\left(\lambda_{0}=\right.$ $2.18 \mu \mathrm{m}, \Delta \lambda=0.3 \mu \mathrm{m}$ ) data reported here were the by-product of a nulling observation (being prepared for a separate publication), and the calibration sequence is somewhat modified from the standard procedures; in particular, only one ratio measurement was made per integration.

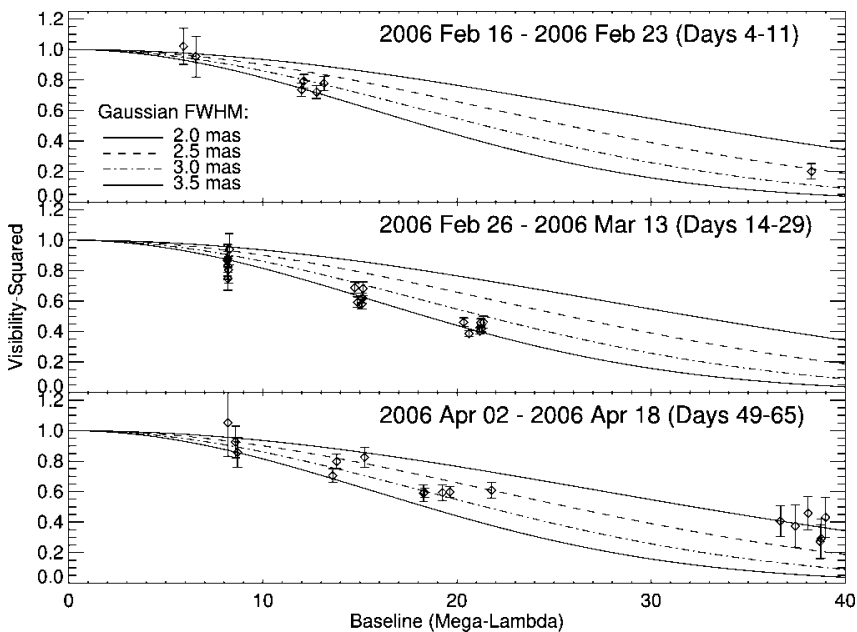

FIG. 1.-UV-averaged visibility data for RS Oph split into three different time periods. The $x$-axis shows the spatial frequency (projected baseline in units of wavelength), while the $y$-axis shows the visibility-squared. Four curves representing Gaussian profiles are also included to show the characteristic size and to allow intercomparison of data in the different panels. For reference, all data shortward of $25 \mathrm{M} \lambda$ derive from IOTA, while longer baseline data come from KI (epoch I) and PTI (epoch III).

Lastly, the Palomar Testbed Interferometer (PTI) observed RS Oph on three nights in 2006 April using the $K$-band detection system $\left(\lambda_{0}=2.20 \mu \mathrm{m}, \Delta \lambda=0.4 \mu \mathrm{m}\right)$ with a $\sim 85 \mathrm{~m}$ baseline (oriented in a northeast direction). Detailed instrument and data analysis descriptions for PTI can be found in the literature (Colavita 1999, 1999). Because of the inherent faintness of the source in April, coherent integration was used for analysis, and a large calibration error of $\Delta V^{2}=0.10$ (absolute, not relative) was added in quadrature with the internal error for model fitting in this Letter.

We have split the data into three epochs-from 2006 February 16 to 23 (days 4-11), from February 26 to March 13 (days 14 29), and from April 2 to April 18 (days 49-65). The UV-averaged visibility data for each epoch are presented in Figure 1, along with some Gaussian profiles for comparison. The IOTA closure phase results are shown in Figure 2, also split into the three epochs. All $V^{2}$ and closure phase data are available from the authors; all data

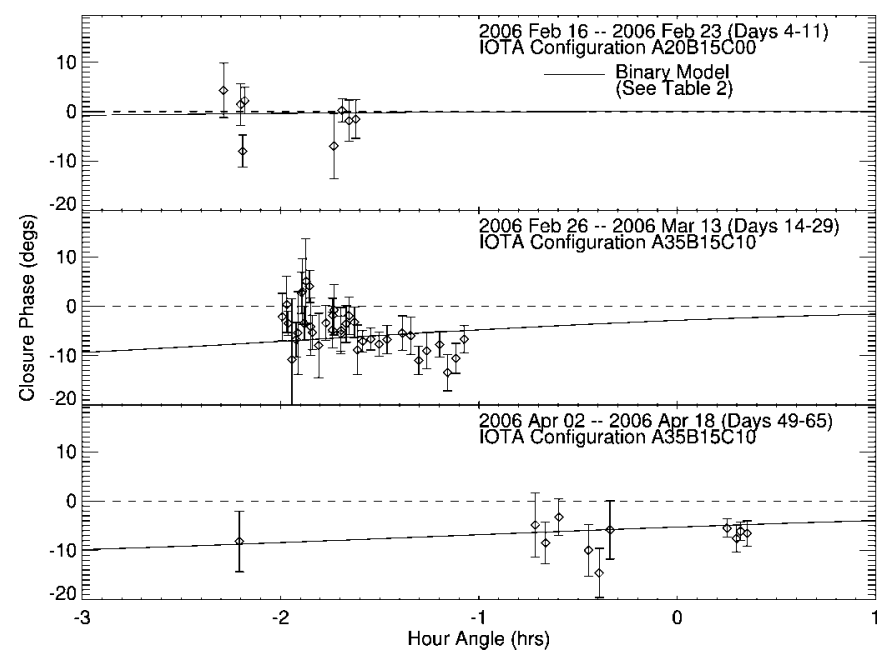

FIG. 2.-IOTA closure phase data for RS Oph split into three different time periods. The $x$-axis shows the hour angle of the observations, while the $y$-axis shows the observed closure phase. The solid line shows the expected closure phase for the binary model parameters found in Table 2 and discussed in $\S 3$. The dashed line shows the expected closure phase signal for the symmetric Gaussian model. 
TABLE 2

RS Oph Model Fitting Results

\begin{tabular}{|c|c|c|c|}
\hline $\begin{array}{l}\text { Model } \\
\text { Parameter }\end{array}$ & $\begin{array}{l}2006 \text { Feb 16-23 } \\
\text { (Days 4-11) }\end{array}$ & $\begin{array}{c}2006 \text { Feb 26-Mar } 13 \\
\text { (Days 14-29) }\end{array}$ & $\begin{array}{l}2006 \text { Apr } 02-18 \\
\text { (Days 49-65) }\end{array}$ \\
\hline \multicolumn{4}{|c|}{ Gaussian Profile (fitting only to $1.65 \mu \mathrm{m}$ ) } \\
\hline $\begin{array}{l}\text { FWHM (mas) } \\
\text { Reduced } \chi^{2}\left(V^{2}\right) \ldots \ldots \ldots \ldots \ldots \\
\text { Reduced } \chi^{2}(\mathrm{CP}) \ldots \ldots \ldots \ldots \ldots \\
\end{array}$ & $\begin{array}{c}3.30 \pm 0.09 \\
0.6 \\
1.3\end{array}$ & $\begin{array}{c}3.47 \pm 0.03 \\
1.3 \\
3.6\end{array}$ & $\begin{array}{c}2.87 \pm 0.07 \\
1.1 \\
5.4\end{array}$ \\
\hline \multicolumn{4}{|c|}{ Gaussian Profile (fitting only to $2.2 \mu \mathrm{m}$ ) } \\
\hline $\begin{array}{l}\text { FWHM (mas) } \ldots \ldots \ldots \ldots \ldots \\
\text { Reduced } \chi^{2}\left(V^{2}\right) \ldots \ldots \ldots \ldots \ldots \\
\text { Reduced } \chi^{2}(\mathrm{CP}) \ldots \ldots \ldots \ldots \ldots\end{array}$ & $\begin{array}{c}2.56 \pm 0.24 \\
0^{\mathrm{a}} \\
\text { N/A }\end{array}$ & $\begin{array}{l}\text { N/A } \\
\text { N/A } \\
\text { N/A }\end{array}$ & $\begin{array}{c}2.00 \pm 0.09 \\
1.0 \\
\text { N/A }\end{array}$ \\
\hline \multicolumn{4}{|c|}{ 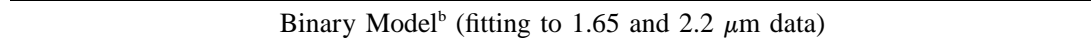 } \\
\hline $\begin{array}{l}\text { Separation (mas) } \ldots \ldots \ldots \ldots \ldots \\
\text { P.A. }{ }^{c}(\text { deg east of north) } \ldots \ldots \ldots \\
\text { Brightness ratio }^{c} \ldots \ldots \ldots \ldots \ldots \ldots \\
\text { Reduced } \chi^{2}\left(V^{2}\right) \ldots \ldots \ldots \ldots \ldots \\
\text { Reduced } \chi^{2}(\mathrm{CP}) \ldots \ldots \ldots \ldots \ldots\end{array}$ & $\begin{array}{l}3.13 \pm 0.12 \\
36 \pm 10^{\mathrm{d}} \\
0.42 \pm 0.06 \\
0.6 \\
1.1\end{array}$ & $\begin{array}{l}3.23 \pm 0.13 \\
45 \pm 5 \\
0.40 \pm 0.06 \\
1.7 \\
1.3\end{array}$ & $\begin{array}{l}3.48 \pm 0.23 \\
27 \pm 5 \\
0.21 \pm 0.03 \\
1.2 \\
0.5\end{array}$ \\
\hline
\end{tabular}

products are stored in the FITS-based, optical interferometry data exchange format (OI-FITS), recently described in Pauls et al. (2005).

\section{ANALYSIS}

Inspection of Figure 1 reveals that the visibility curve for RS Oph changes very little between day 4 and 65 (since the outburst). This is surprising since the $H$-band brightness faded by a factor of $>10$ during this time. This result is discordant with the generic prediction of Evans et al. (1988), who interpreted the IR light curve in terms of the time evolution of postshock gas at $10^{5} \mathrm{~K}$ moving at the speed of the contact discontinuity, $1400 \mathrm{~km} \mathrm{~s}^{-1}$ (following the earlier work by Bode \& Kahn 1985). This model requires the IR emission to be seen expanding at a rate of $\sim 1.0$ mas day ${ }^{-1}$ (assuming a distance of $1.6 \mathrm{kpc})$, an interpretation that is now ruled out.

Before discussing alternative models in $\S 4$, we wish to carry out some model fitting to the interferometry data. Here we only consider two simple models-a circularly symmetric Gaussian and a binary star model. For all fits and calculation of reduced $\chi^{2}$, we have used the original data points before UV averaging.

First, we fit a circularly symmetric Gaussian to each epoch of data, split by wavelength. Table 2 contains the best-fitting FWHM and the reduced $\chi^{2}$ for both the $V^{2}$ and CP (the model $\mathrm{CP}$ is always zero for a Gaussian profile). The Gaussian model is a reasonable fit for the IOTA visibility alone but clearly cannot fit the nonzero closure phase seen in March and April. Also, Figure 1 shows that no good fit was possible when combining IOTA with Keck and PTI data, indicating that this model is too simplistic to explain the full range of baselines and/or the wavelength dependence. Sizes derived from the longer baseline $K$-band data are systematically smaller than those derived from shorter baseline $H$-band data (IOTA).

As discussed in $\S 1$, Hachisu \& Kato (2001) suggest that the nova's IR light curve might be due primarily to a rapid increase in brightness of the WD as it returns, albeit briefly, to a red giant phase. Motivated by this work, we realized that the IR emission might be due to the underlying RS Oph binary itself, and this might explain the general puzzling features of our data: nonexpanding emission size, the inadequacies of the Gaussian fit, and the nonzero closure phase.
In order to test this idea, we fit binary models to the data for each of the three time periods, treating the brightness ratio as independent of wavelength in order to fit the $H$ - and $K$-band data together. The IOTA, KI, and PTI complement each other in Fourier coverage, and an exhaustive grid search of separations less than 10 mas found unique binary star solutions. ${ }^{13}$

Table 2 contains the best-fit binary models for the three time periods, including the reduced $\chi^{2}\left(V^{2}, \mathrm{CP}\right)$. All three epochs are reasonably fit by a similar binary model. The only parameter that changed significantly between the epochs was the brightness ratio. The closure phase predictions for the binary models are plotted along with the closure phase data in Figure 2. We note that our $(u, v)$-plane is missing coverage in the northwest direction, and thus elongated structure in this direction would be observed foreshortened.

\section{DISCUSSION}

Because the expanding fireball model fails to explain the nearly static size scale of the IR emission, we now seek suitable alternative emission mechanisms for the time-variable IR emission from the recurrent nova RS Oph. We have pursued the reborn red giant (thermonuclear runaway) model of Hachisu \& Kato (2001) and found that indeed our three-interferometer combined data set can be explained by a simple binary model with separation of $\sim 3.2$ mas, P.A. $30^{\circ}$ east of north, and a brightness ratio varying from $2.5: 1$ to $5: 1$. Next we subject the binary hypothesis to further scrutiny.

\subsection{Binary Interpretation of Near-Interferometry Data}

On the basis of single-line radial velocity data, Fekel et al. (2000) find the RS Oph binary orbit to be roughly circular with a period of $455.72 \pm 0.83$ days and mass function $f=$ $0.221 \pm 0.038 M_{\odot}$. RGs in symbiotic systems are typically $1-$ $3 M_{\odot}$ (Dobrzycka \& Kenyon 1994), and we expect recurrent novae to contain a Chandrasekhar mass WD $\left(1.4 M_{\odot}\right)$; these facts combined with the known mass function rule out RG masses greater than $2 M_{\odot}$. Assuming the RS Oph system mass

\footnotetext{
${ }^{13}$ Our limited $(u, v)$-coverage admits some unlikely additional solutions with larger binary separations, which will be discussed fully in a future modeling paper.
} 
to be 2.4-3.4 $M_{\odot}$, we find the component separation to be 1.55-1.74 AU, or (unprojected) 2.59-2.90 mas at the $600 \mathrm{pc}$ distance preferred by Hachisu \& Kato (2001) — only slightly smaller than our observed separation of 3.2 mas.

Since the RS Oph outburst took place only 1 month before maximum redshifted velocity (Fekel et al. 2000), our measured binary parameters represent the true orbital semimajor axis and orbital $\Omega$ for RS Oph with only weak sin $i$ dependencies. Thus, a small reduction in the distance estimate $(540 \mathrm{pc})$ brings the interferometer binary model in agreement with expectations from Kepler's laws.

The binary model fits (Table 2) show evidence for a change in the brightness ratio over time. While the Hachisu \& Kato (2001) theory predicts a time-changing brightness ratio, it is beyond the scope of this Letter to test the compatibility with the observed IR light curves due to complications from the role of the irradiated $\mathrm{RG}$ photosphere and the presence of a postoutburst WD accretion disk.

\subsection{Circumbinary Reservoir of Hot Gas}

The distance estimate of $\$ 540 \mathrm{pc}$ derived in the last section stands in strong contrast to estimates more commonly adopted in the literature. The most significant constraints on distance are set by resolved radio observations of the previous and current burst (Hjellming et al. 1986; Taylor et al. 1989; M. Rupen, A. J. Mioduszewski, \& M. Sokoloski 2006, in preparation; O'Brien et al. 2006). By assuming that observed radio proper motions (on the sky) can be ascribed to the fast-moving ejecta or forward shock, workers consistently derive a distance of $\sim 1600$ pc. Similarly, large distances were found considering interstellar UV absorption lines (Snijders 1987) and H I absorption measurements (Hjellming et al. 1986).

Given the strength of the evidence, we now consider the implications of the $d=1600 \mathrm{pc}$ distance. This distance would rule out the binary interpretation of the near-IR interferometry data laid out in $\S 4.1$, given existing binary constraints.

Instead, we hypothesize that the IR emission arises from a quasistationary ${ }^{14}$ hot gas reservoir that contributes a combination of emission lines and free-free/bound-free emission in the NIR bands. The observed FWHM of $\sim 3$ mas is $\sim 5 \mathrm{AU}$ at 1600 pc, about 3

\footnotetext{
${ }^{14}$ It is possible to fit our data with an expanding wind or jet component, but this requires finetuning the relative proportions of multiple components and/or a very asymmetric jetlike emission oriented perpendicular to our long (northeast) baselines. These possibilities will be investigated more thoroughly in future work with an extended data set.
}

times the expected RS Oph binary separation. This size is reasonable for a circumbinary disk or reservoir of hot gas, perhaps kept ionized by the outward moving blast wave or soft X-ray luminosity from the WD itself following outburst. This gas reservoir might be analogous to the "fallback disk" inferred to form after some supernovae (e.g., Wang et al. 2006).

Clearly, the hypothesized gas reservoir must be elongated and somewhat off-center with respect to the central source in order to fit the combined IOTA, KI, and PTI interferometry data, especially the nonzero closure phases. It is beyond the scope of this Letter to investigate the details here, and we defer development of this model to a future paper.

\subsection{Future Work}

While we have ruled out the important class of expanding fireball models for explaining the IR emission from the recurrent nova RS Oph, more work lies ahead to test the other emission mechanisms discussed in this Letter. A future study will attempt to synthesize a self-consistent model that can explain the time evolution of the IR spectrum, NIR and mid-IR interferometer data, and multiwavelength light curves at the same time.

If the close distance $d \lesssim 540$ pc is confirmed, we have a spectacular opportunity to study in detail a likely SN Ia progenitor and to learn about unexpected shock physics controlling the nonthermal radio emission. Alternatively, the farther distance $d \sim 1600 \mathrm{pc}$ suggests we have discovered a significant and new component to the RS Oph Nova remnant, and future work will characterize the hot circumbinary gas reservoir for the first time.

Note added in manuscript.-Future modeling should address the asymmetric radio nebula and jet observed by O'Brien et al. (2006).

We thank J. Sokoloski for productive discussion and comments on the manuscript and members of the AAVSO IR group for early-time photometry. R. K. B. acknowledges NASA and Dr. Bill Danchi for supporting this work. We acknowledge SAO, NSF, NASA, CNRS/CNES (France), LAOG, IMEP, and LETI for support of IOTA and IONIC3. KI was developed and is operated by JPL, MSC, and WMKO with funding from NASA. PTI was developed by JPL and is operated by the MSC on behalf of the PTI collaboration. This research made use of SIMBAD, ADS, MSC resources, CHARM2, and the 2MASS catalog. Some data presented herein were obtained at the Keck Observatory, operated by a scientific partnership among Caltech, UC, and NASA.

\section{REFERENCES}

Berger, J.-P., et al. 2003, Proc. SPIE, 4838, 1099

Bode, M. F., \& Kahn, F. D. 1985, MNRAS, 217, 205

Bordé, P., Coudé du Foresto, V., Chagnon, G., \& Perrin, G. 2002, A\&A, 393, 183

Cohen, M., Walker, R. G., Carter, B., Hammersley, P., Kidger, M., \& Noguchi, K. 1999, AJ, 117, 1864

Colavita, M. M. 1999, PASP, 111, 111

Colavita, M. M., \& Wizinowich, P. L. 2000, Proc. SPIE, 4006, 310 2003, Proc. SPIE, 4838, 79

Colavita, M. M., et al. 1999, ApJ, 510, 505 2003, ApJ, 592, L83

Dobrzycka, D., \& Kenyon, S. J. 1994, AJ, 108, 2259

Evans, A., Callus, C. M., Albinson, J. S., Whitelock, P. A., Glass, I. S., Carter, B., \& Roberts, G. 1988, MNRAS, 234, 755

Fekel, F. C., Joyce, R. R., Hinkle, K. H., \& Skrutskie, M. F. 2000, AJ, 119, 1375

Gehrz, R. D. 1988, ARA\&A, 26, 377

Hachisu, I., \& Kato, M. 2001, ApJ, 558, 323

Hjellming, R. M., van Gorkom, J. H., Seaquist, E. R., Taylor, A. R., Padin, S., Davis, R. J., \& Bode, M. F. 1986, ApJ, 305, L71
Kato, M. 1991, ApJ, 369, 471

Lane, B. F., Retter, A., Eisner, J. A., Thompson, R. R., \& Muterspaugh, M. W. 2005, BAAS, 37, 1335

Monnier, J. D., et al. 2004, ApJ, 602, L57 2006, ApJ, 647, 444

Narumi, H., Hirosawa, K., Kanai, K., Renz, W., Pereira, A., Nakano, S., Nakamura, Y., \& Pojmanski, G. 2006, IAU Circ., 8671, 2

O'Brien, T. J., et al. 2006, Nature, 442, 279

Pauls, T. A., Young, J. S., Cotton, W. D., \& Monnier, J. D. 2005, PASP, 117, 1255

Snijders, M. A. J. 1987, in RS Ophiuchi (1985) and the Recurrent Nova Phenomenon, ed. M. F. Bode (Utrecht: VNU), 51

Taylor, A. R., Davis, R. J., Porcas, R. W., \& Bode, M. F. 1989, MNRAS, 237, 81

Traub, W. A., et al. 2003, Proc. SPIE, 4838, 45

van Belle, G. T., et al. 1999, AJ, 117, 521

Wang, Z., Chakrabarty, D., \& Kaplan, D. L. 2006, Nature, 440, 772

Warner, B. 1976, in IAU Symp. 73, Structure and Evolution of Close Binary Systems, ed. P. Eggleton, S. Mitton, \& J. Whelan (Dordrecht: Reidel), 85 